\title{
Tratamento cirúrgico das fraturas supra e intercondilianas do úmero
}

\author{
Surgical treatment of supra and intercondilar humeral fractures
}

Roberto Yukio Ikemoto ${ }^{1,2}$, Joel Murachovsky ${ }^{2}$, Luis Gustavo Prata Nascimento², Rogerio Serpone Bueno², Luis Henrique Almeida², Eric Strose'2, Paulo Bonadio Telles ${ }^{2}$

\section{Resumo}

Introdução: As fraturas supra e intercondilianas do úmero, em adultos, são lesões complexas e de difícil tratamento, pelas características anatômicas locais e pela grande fragmentação óssea que geralmente ocorre. Objetivo: Avaliar o resultado funcional do tratamento cirúrgico dessas fraturas em adultos. Método: No período de junho de 1999 a agosto de 2005, 22 pacientes (22 cotovelos) com idade entre 20 e 70 anos, com fraturas supra e intercondilianas do úmero, foram submetidos a tratamento cirúrgico mediante redução aberta e fixação interna rígida, com placas e parafusos de 3,5 mm, por via de acesso posterior. Resultados: De acordo com o critério de Jupter et al, obtivemos 68,2\% de resultados classificados como satisfatórios e, como complicações do tratamento, um paciente $(4,5 \%)$ apresentou neuropraxia ulnar, que regrediu espontaneamente e outros dois (9\%) que apresentavam dor no olécrano, causada pelo material de síntese. Após a retirada desse material de síntese referiram melhora. Conclusões: 0 tratamento cirúrgico para as fraturas supra e intercondilianas do úmero mostrou ser um método eficaz com $68,2 \%$ de resultados classificados como satisfatórios neste estudo.

Palavras-chave: Fraturas do úmero; cotovelo; resultado de tratamento; cirurgia.

\section{Abstract}

Introduction: The supracondylar and intercondylar humerus fractures are complex injuries of difficult treatment because of the local anatomic features and the bone fragmentation that generally occurs. Objective: To access the functional outcome of surgical treatment of these fractures in adults. Method: From June 1999 to August 2005, 22 patients (22 elbows) ranging from 20 to 70 years old who had supracondylar and intercondylar humerus fractures were submitted to surgical treatment by posterior approach with open reduction and internal fixation with $3,5 \mathrm{~mm}$ screws and plates. Results: According to Jupiter's criteria, we found satisfactory results in $68.2 \%$ of the patients. One patient $(4,5 \%)$ presented ulnar neuropraxia with spontaneous subsidence, and two patients ( $9 \%$ ) who had pain caused by the tension band on the olecranon needed to have it removed and then were satisfied with the result. Conclusions: In this study, the surgical treatment for the supracondylar and intercondylar humerus fractures led to an acceptable rate of satisfactory outcomes $(68,2 \%)$.

Keywords: Humeral fractures; elbow; treatment outcome; surgery.

Recebido: 5/10/2009

Revisado: $19 / 11 / 2009$

Aprovado: $12 / 1 / 2010$

\footnotetext{
Trabalho realizado no Hospital Estadual Ipiranga - São Paulo (SP), Brasil

Departamento de Ortopedia e Traumatologia do Hospital Estadual Ipiranga, São Paulo (SP), Brasil

2 Grupo de Cirurgia do Ombro e Cotovelo da Faculdade de Medicina do ABC e do Hospital Ipiranga, São Paulo (SP), Brasil

Endereço para correspondência: Rogerio Serpone Bueno - Serviço de Ortopedia do Hospital Mário Covas - Rua Doutor Henrique Calderazzo, 321 -

CEP 09190-615 - Santo André (SP) - E-mail: rsbueno@hotmail.com
} 


\section{Introdução}

As fraturas supra e intercondilianas do úmero, descritas originalmente por Desault, em $1811^{1}$, são incomuns e correspondem a $2 \%$ de todas as fraturas do adulto e a $1 / 3$ das fraturas do úmero ${ }^{2}$. São fraturas de fácil diagnóstico com base na clínica e no estudo radiográfico ${ }^{3}$. Geralmente, são causadas por trauma direto no olécrano e, menos frequentemente, por traumas indiretos com cotovelo em hiperextensão ${ }^{4}$.

O úmero distal apresenta duas colunas ósseas, uma medial e outra lateral, as quais são separadas pela fossa do olécrano e mais distalmente pela tróclea. A superfície articular anterolateral é composta pelo capítulo e a medial pela tróclea. Nas fraturas do terço distal do úmero as origens musculares provocam desvios rotacionais dos fragmentos, tornando-se difícil a redução incruenta ${ }^{5}$.

As fraturas intra-articulares do terço distal do úmero consistem em lesões graves que provocam incapacidade funcional do membro afetado, sendo que o tratamento dessas fraturas tem sido debatido por décadas 5 . No passado, o uso de redução incruenta e de imobilização era considerado o tratamento de escolha, devido aos maus resultados com o tratamento cirúrgico ${ }^{6}$. A partir da introdução, pelo grupo $\mathrm{AO} /$ ASIF, dos métodos de fixação interna e de reconstrução articular, muitos autores têm demonstrado resultados satisfatórios com o tratamento cirúrgico combinado com a mobilização precoce do cotovelo, mesmo em fraturas multifragmentares intra-articulares ${ }^{7,8}$.

As complicações mais frequentemente encontradas após procedimento cirúrgico são: infecção, falha da síntese, retardo de consolidação, pseudoartrose, lesões neurovasculares, ossificação heterotópica e osteoartrite pós-traumática ${ }^{3,4,5}$. Assim, o objetivo deste trabalho foi analisar os resultados funcionais do tratamento cirúrgico das fraturas supra e intercondilianas do úmero.

\section{Método}

Entre junho de 1999 e agosto de 2005, 22 fraturas do terço distal do úmero em 22 pacientes, 15 do sexo masculino e 7 do feminino, foram tratadas por meio de redução aberta e fixação interna. A média de idade entre os pacientes operados foi de 37 anos (variando de 20 a 70 anos). A média do seguimento foi de 32 meses (variando de 12 a 69 meses) e, em média, o período decorrente entre trauma e procedimento cirúrgico foi de 7 dias (variando de 2 a 22 dias). O lado esquerdo foi o mais acometido (14 casos $-63,6 \%$ ).

Quanto às causas que levaram à fratura, cinco pacientes foram vítimas de acidente automobilístico, outros cinco de atropelamento, três sofreram ferimentos de arma de fogo e nove, quedas ao solo. Dois casos apresentaram lesões associadas, sendo uma fratura do colo do fêmur ipsilateral e lesão neurológica do nervo radial e ulnar no exame físico de entrada.

Foram avaliadas as mobilidades de flexão, extensão, pronação e supinação dos cotovelos submetidos à cirurgia. Avaliamos os resulta- dos pela escala de Júpiter et al. ${ }^{9}$ e comparamos as mobilidades de flexão e extensão de acordo com o tipo de fratura da classificação $\mathrm{AO} / \mathrm{ASIF}^{8}$ : duas fraturas tipo A2, duas A3, três $\mathrm{C} 1$ e $15 \mathrm{C} 2$. Houve três casos de fratura exposta, classificadas como tipo II, de Gustillo e Andersen ${ }^{10}$ (Tabela I).

Calcularam-se as medidas de posição (médias, medianas, frequências e porcentagens) e medidas de dispersão (desvio padrão, mínimo e máximo). Utilizamos o teste de Mann-Whitney para a análise estatística dos resultados ${ }^{11}$. Os testes foram realizados com nível de significância a 5\%.

Este estudo foi aprovado no Comitê de Ética em Pesquisa da Faculdade de Medicina do ABC sob o número 280/2007.

\section{Técnica cirúrgica}

O procedimento foi realizado por meio de via de acesso posterior com o paciente em decúbito ventral, sob anestesia geral. O nervo ulnar foi rotineiramente identificado e isolado. Em 18 pacientes foi realizada osteotomia em "V" do olécrano, do tipo chevron, para a exposição da articulação e, nos demais casos, o acesso foi paratriciptal.

A fixação interna foi realizada seguindo dois passos: (1) reconstrução anatômica da articulação, utilizando fios de Kirschner e colocação de parafusos de osso esponjoso de $3,5 \mathrm{~mm}$; (2) restabelecimento da integridade da superfície articular, realizando sua redução com a metáfise e posterior osteossíntese com placa de reconstrução acetabular ou DCP de 3,5 mm na região posterior da coluna lateral e terço tubular na medial.

A osteotomia do olécrano, quando realizada, foi fixada com dois fios de Kirschner de $2 \mathrm{~mm}$ em associação à cerclagem com fio de aço com 1,2 mm de diâmetro, utilizando o princípio de banda de tensão.

Em nenhum caso foi utilizada imobilização gessada no pós-operatório, dessa forma, foi iniciada a fisioterapia (exercícios isométricos e mobilização passiva) 48 horas após o tratamento cirúrgico.

\section{Resultados}

O intervalo de tempo entre o trauma e a realização do tratamento cirúrgico foi em média de 7 dias, com mínimo de 2 e máximo de 22 dias.

Houve consolidação de todas as fraturas e osteotomias do olécrano. Entretanto, dois pacientes submetidos à osteotomia queixavam-se de dor no local da síntese mesmo após consolidação, optando-se, assim, pela retirada do material de síntese, o que proporcionou a melhora do quadro álgico.

Observou-se que a média de flexão obtida foi de $124^{\circ}$ (variando de $100^{\circ}$ a $140^{\circ}$ ) e que 8 pacientes apresentavam flexão acima da média referida. Quanto à extensão, a média obtida foi de $-17^{\circ}$ (variando de $0^{\circ}$ a $-40^{\circ}$ ) e 12 pacientes apresentavam extensão acima da média. Quanto ao arco de movimento pós-operatório (flexoextensão), a média foi de $107^{\circ}$ 
(variando de $70^{\circ}$ a $140^{\circ}$ ). Já o arco de movimento de pronossupinação foi de $172^{\circ}$ (variando $160^{\circ}$ a $180^{\circ}$ ) (Tabela II).

A Tabela III apresenta as comparações da flexão e da extensão entre os pacientes com lesão grave e menos grave. Como complicação, observou-se a neuropraxia pós-operatória em um caso, excetuando a paralisia do nervo ulnar e radial em um paciente, já diagnosticada no exame pré-operatório.

Nas avaliações finais, utilizando a escala de Jupiter et al. ${ }^{9}$, obtivemos $27,27 \%$ dos casos com resultados excelentes, $40,9 \%$ bons, $27,27 \%$ regulares e apenas $4,54 \%$ ruins ( 1 caso).

Tabela I - Pacientes com fraturas supra e intercondilianas tratados cirurgicamente

\begin{tabular}{|c|c|c|c|c|c|c|c|c|c|c|c|}
\hline $\begin{array}{l}\text { Número } \\
\text { do Caso }\end{array}$ & $\begin{array}{c}\text { Idade } \\
\text { (em anos) }\end{array}$ & Sexo & Trauma & $\begin{array}{c}\text { Lado } \\
\text { acometido }\end{array}$ & $\begin{array}{c}\text { Classificação } \\
\text { A0/ASIF }\end{array}$ & $\begin{array}{l}\text { Osteotomia } \\
\text { do olécrano }\end{array}$ & $\begin{array}{c}\text { Tempo do } \\
\text { trauma à } \\
\text { cirurgia } \\
\text { (em dias) }\end{array}$ & $\begin{array}{l}\text { Seguimento } \\
\text { (em meses) }\end{array}$ & $\begin{array}{c}\text { Mobilidade } \\
\text { (flexão/extensão/ } \\
\text { pronação/ } \\
\text { supinação) }\end{array}$ & $\begin{array}{l}\text { Complicações no } \\
\text { Pós-operatório }\end{array}$ & $\begin{array}{c}\text { Resultados } \\
\text { na Escala } \\
\text { de Jupiter }\end{array}$ \\
\hline 1 & 22 & $\mathrm{M}$ & FAF & $E$ & $\mathrm{C} 2$ & + & 5 & 12 & $\begin{array}{c}110^{\circ} /-40^{\circ} \\
90^{\circ} / 90^{\circ}\end{array}$ & Dor & Ruim \\
\hline 2 & 36 & $\mathrm{~F}$ & QS & $\mathrm{D}$ & $\mathrm{C} 2$ & + & 12 & 18 & $\begin{array}{c}110^{\circ} /-30^{\circ} \\
90^{\circ} / 90^{\circ}\end{array}$ & $*$ & Regular \\
\hline 3 & 39 & M & ATR & $\mathrm{D}$ & $\mathrm{C} 2$ & + & 4 & 44 & $\begin{array}{c}100^{\circ} /-25^{\circ} \\
90^{\circ} / 80^{\circ}\end{array}$ & * & Regular \\
\hline 4 & 44 & $\mathrm{M}$ & FAF & $\mathrm{D}$ & A3 & - & 6 & 60 & $\begin{array}{c}130^{\circ} /-25 \\
80^{\circ} / 80^{\circ}\end{array}$ & * & Bom \\
\hline 5 & 24 & $\mathrm{M}$ & AA & $E$ & A2 & - & 5 & 16 & $\begin{array}{l}140^{\circ} / 0^{\circ} \\
90^{\circ} / 90^{\circ}\end{array}$ & * & Excelente \\
\hline 6 & 70 & $\mathrm{~F}$ & QS & $\mathrm{E}$ & $\mathrm{C} 2$ & + & 3 & 61 & $\begin{array}{c}125^{\circ} /-10^{\circ} \\
80^{\circ} / 90^{\circ}\end{array}$ & Parestesia no nervo ulnar & Bom \\
\hline 7 & 49 & $\mathrm{~F}$ & OS & $\mathrm{E}$ & $\mathrm{C} 2$ & + & 7 & 30 & $\begin{array}{l}135^{\circ} / 5^{\circ} \\
80^{\circ} / 80^{\circ}\end{array}$ & * & Excelente \\
\hline 8 & 51 & $\mathrm{M}$ & $A A$ & $E$ & $\mathrm{C} 1$ & + & 6 & 24 & $\begin{array}{c}135^{\circ} /-10^{\circ} \\
90^{\circ} / 90^{\circ}\end{array}$ & $*$ & Excelente \\
\hline 9 & 26 & $\mathrm{~F}$ & ATR & E & C1 & + & 2 & 36 & $\begin{array}{c}125^{\circ} /-25^{\circ} \\
80^{\circ} / 90^{\circ}\end{array}$ & Dor & Bom \\
\hline 10 & 44 & $\mathrm{M}$ & ATR & D & C1 & + & 4 & 48 & $\begin{array}{c}130^{\circ} /-10^{\circ} \\
90^{\circ} / 90^{\circ}\end{array}$ & $*$ & Regular \\
\hline 11 & 25 & $\mathrm{M}$ & FAF & $\mathrm{E}$ & A3 & + & 7 & 24 & $\begin{array}{c}130^{\circ} /-10^{\circ} \\
80^{\circ} / 90^{\circ}\end{array}$ & * & Bom \\
\hline 12 & 43 & $\mathrm{M}$ & AA & $D$ & A2 & - & 6 & 69 & $\begin{array}{c}130^{\circ} /-10^{\circ} \\
90^{\circ} / 90^{\circ}\end{array}$ & $*$ & Bom \\
\hline 13 & 20 & $M$ & $A A$ & D & $\mathrm{C} 2$ & + & 3 & 48 & $\begin{array}{c}130^{\circ} /-30^{\circ} \\
90^{\circ} / 80^{\circ}\end{array}$ & * & Bom \\
\hline 14 & 65 & $\mathrm{~F}$ & OS & D & $\mathrm{C} 2$ & + & 4 & 28 & $\begin{array}{c}140^{\circ} /-15^{\circ} \\
80^{\circ} / 80^{\circ}\end{array}$ & $*$ & Excelente \\
\hline 15 & 26 & $M$ & $A A$ & $E$ & $\mathrm{C} 2$ & + & 22 & 20 & $\begin{array}{c}100^{\circ} /-30^{\circ} \\
90^{\circ} / 90^{\circ}\end{array}$ & $*$ & Regular \\
\hline 16 & 38 & $M$ & ATR & D & $\mathrm{C} 2$ & + & 3 & 43 & $\begin{array}{c}120^{\circ} / 0 \\
80^{\circ} / 80^{\circ}\end{array}$ & * & Bom \\
\hline 17 & 35 & $M$ & QS & $\mathrm{E}$ & $\mathrm{C} 2$ & + & 15 & 30 & $\begin{array}{c}110^{\circ} /-30^{\circ} \\
80^{\circ} / 80^{\circ}\end{array}$ & * & Regular \\
\hline 18 & 29 & $\mathrm{M}$ & QS & $E$ & $\mathrm{C} 2$ & + & 5 & 12 & $\begin{array}{c}120^{\circ} /-15^{\circ} \\
90^{\circ} / 90^{\circ}\end{array}$ & $*$ & Bom \\
\hline 19 & 42 & $M$ & ATR & $E$ & $\mathrm{C} 2$ & + & 16 & 17 & $\begin{array}{l}140^{\circ} / 0 \\
90^{\circ} / 90^{\circ}\end{array}$ & * & Excelente \\
\hline 20 & 24 & $\mathrm{~F}$ & QS & $E$ & $\mathrm{C} 2$ & + & 4 & 18 & $\begin{array}{c}125^{\circ} /-20^{\circ} \\
80^{\circ} / 90^{\circ}\end{array}$ & $*$ & Bom \\
\hline 21 & 32 & $\mathrm{~F}$ & QS & $\mathrm{E}$ & $\mathrm{C} 2$ & - & 3 & 15 & $\begin{array}{c}110^{\circ} /-30^{\circ} \\
80^{\circ} / 80^{\circ}\end{array}$ & $*$ & Regular \\
\hline 22 & 23 & $\mathrm{M}$ & QS & $E$ & $\mathrm{C} 2$ & + & 4 & 27 & $\begin{array}{c}140^{\circ} /-10^{\circ} \\
85^{\circ} / 90^{\circ}\end{array}$ & $*$ & Excelente \\
\hline
\end{tabular}

M: masculino; F: feminino, FAF: ferimento por arma de fogo; QS: queda ao solo; ATR: atropelamento; AA: acidente automobilístico. 
Tabela II - Descrição das medidas numéricas quanto ao arco de movimento

\begin{tabular}{lcrrccc}
\hline & N & Média & DP & Mínimo & Máximo & Mediana \\
\hline Flexão & 22 & 124,32 & 12,75 & 100 & 140 & 127,5 \\
Extensão & 22 & $-17,27$ & 11,72 & -40 & 0 & -15 \\
Pronação & 22 & 85,23 & 4,99 & 80 & 90 & 87,5 \\
Supinação & 22 & 86,36 & 4,92 & 80 & 90 & 90 \\
\hline
\end{tabular}

DP: desvio padrão / N: número de pacientes.

Tabela III - Comparação entre a classificação das fraturas e a amplitude articular

\begin{tabular}{lccccc}
\hline Variável & $\begin{array}{c}\text { Classificação } \\
\text { A0/ASIF }\end{array}$ & $n$ & Média & DP & $\begin{array}{c}\text { Significância } \\
\text { (p) }\end{array}$ \\
\hline Mobilidade Flexão & $\mathrm{A}$ & 4 & 132.50 & 5 & 0,018 \\
& $\mathrm{C}$ & 18 & 122.50 & 13,31 & \\
Mobilidade Extensão & $\mathrm{A}$ & 4 & -11.25 & 10,31 & \multirow{2}{*}{0,047} \\
& $\mathrm{C}$ & 18 & -18.61 & 11,86 & \\
\hline
\end{tabular}

n: número de pacientes; DP: desvio padrão; p: índice de significância.

Tabela IV - Resultado do tratamento das fraturas Supra e intercondilianas do úmero

\begin{tabular}{|c|c|c|c|}
\hline Autor & Ano & Excelente/Bom (\%) & Regular/Ruim (\%) \\
\hline Jupiter et $a l .^{9}$ & 1985 & 79 & 21 \\
\hline Widberger et al.* & 1991 & 89 & 11 \\
\hline John et al.* & 1994 & 85 & 15 \\
\hline Wong et al.* & 1994 & 75 & 25 \\
\hline Kundel et al. ${ }^{4}$ & 1996 & 52 & 48 \\
\hline Pajarinen e Bjorkenheim ${ }^{12}$ & 2002 & 56 & 44 \\
\hline Checchia et al. ${ }^{7}$ & 2003 & 69 & 31 \\
\hline Neste estudo & 2005 & 68 & 32 \\
\hline
\end{tabular}

${ }^{*}$ Fonte: Kundel et $a /{ }^{4}$

\section{Discussão}

As fraturas supra e intercondilianas do úmero necessitam de tratamento cirúrgico, objetivando-se redução anatômica e estabilidade absoluta articular para promover a reabilitação precoce do paciente às suas atividades, evitando a perda do arco de movimento da articulação do cotovelo ${ }^{1,3,6,9,12,13}$.

O mecanismo de trauma mais comum na maioria dos estudos é a queda ao solo ${ }^{1,2,7,14-16}$, mas em nossa casuística predominou o acidente automobilístico, o que também é relatado por outros autores $^{12,16,17}$.

O tempo decorrido entre o trauma e a realização do tratamento cirúrgico foi em média de sete dias. Os determinantes para a decisão do melhor momento para a intervenção cirúrgica foram: o edema, as condições de pele e a ausência de sinais de infecção nos casos de fraturas expostas. Segundo Kundel et $a l .^{4}$, as fraturas tratadas com menos de 24 horas de evolução apresentam melhor prognóstico quanto ao arco de movimento e diminuição de ossificação heterotópica, porém não foi encontrada diferença significativa no resultado final quando comparado o tempo decorrido entre o trauma e a cirurgia.
A osteotomia do olécrano realizada por acesso posterior, preconizada por Cassebaum ${ }^{18}$, permite ampla exposição da região articular do úmero distal, tornando possível a redução anatômica dos possíveis fragmentos ósseos. Comparando-se as vias transtriciptais às paratriciptais, nota-se que aquelas expõem melhor a superfície articular, podendo-se visibilizar todo o capítulo e a troclea ${ }^{19}$.

Já a osteotomia em "V", tipo chevron, apresenta as vantagens de facilitar a redução da osteotomia, sua fixação e de aumentar a área de contato ósseo, estimulando sua consolidação sem comprometer o resultado funcional do cotovelo. Alguns autores evidenciaram resultados similares ao comparar o acesso transtriciptal com a osteotomia do olécrano, nos casos de fraturas extra-articulares ou articulares simples ${ }^{3,12,18}$.

Em nosso estudo, foi realizada a osteotomia do olécrano em 18 pacientes, 17 com fraturas articulares (tipo C2) e 1 paciente com fratura extra-articular multifragmentar (tipo A3) por ferimento de arma de fogo; nesse, a cirurgia foi realizada para facilitar a redução da fratura. Em todos os casos houve consolidação da osteotomia, embora a literatura apresente uma incidência de até 7\% de casos que evoluíram para pseudoartrose ${ }^{1,18,20}$.

Em quatro pacientes não foi necessária a realização da osteotomia. Em três deles, por serem fraturas extra-articulares (dois tipo A2 e um tipo A3) e, em outro caso, por ser uma fratura com traço simples na superfície articular (tipo C2). Em todos, foi possível a redução indireta sob radioescopia.

Estudos biomecânicos mostram que a estabilidade absoluta é alcançada quando a fixação das duas colunas é realizada de maneira ortogonal ${ }^{4,21}$, sendo este o método escolhido para a fixação de todas as fraturas neste estudo.

Para a avaliação dos resultados do tratamento cirúrgico, utilizamos a escala funcional de Jupiter et al. ${ }^{9}$, que classifica os resultados pósoperatórios em excelente, bom, regular e ruim, sendo considerados satisfatórios os casos classificados como excelentes e bons $\mathrm{s}^{4,9,15,16}$. Essa escala foi escolhida por ser utilizada pela maioria dos autores para avaliar seus resultados, o que possibilita a comparação de nossos resultados com os dados da literatura.

Por meio do tratamento cirúrgico, é possível obter, na maioria das vezes, resultados satisfatórios. A Tabela IV mostra a porcentagem de resultados classificados como satisfatórios alcançados por diversos autores que utilizaram o mesmo critério de avaliação usado neste estudo. A variação foi de 52 a $89 \%$ de excelentes e bons resultados.

Em aproximadamente $32 \%$ dos casos deste estudo, os resultados foram classificados como insatisfatórios. Tal proporção assemelha-se ao encontrado em outros estudos ${ }^{1,4,9,15,16,22}$. As principais causas que levam a resultados funcionais ruins são: fraturas expostas, fraturas multifragmentares que comprometam a articulação (tipo C da classificação AO/ ASIF), outro trauma importante no mesmo membro e politrauma ${ }^{4,16}$. Para alguns autores, a idade acima de 50 anos é citada como um fator predisponente para maus resultados ${ }^{16}$. Porém, em muitos estudos, inclusive na nossa série, não houve correlação entre a idade avançada $\mathrm{e}$ os maus resultados ${ }^{1,4,14,15,17}$. 
Diversos estudos mostram que a fratura supra e intercondiliana do úmero leva a uma limitação do arco de movimento, mesmo após o tratamento cirúrgico ${ }^{9,12,13,15,16}$. Obtivemos como resultado um arco de movimento médio de $108^{\circ}$, semelhante aos resultados obtidos em outras séries ${ }^{9,12,13,15,16}$. Segundo Morrey et al. ${ }^{17}$, o arco de movimento a partir de $100^{\circ}$, é considerado funcional para as atividades diárias.

Como complicações, um paciente $(4,5 \%)$ apresentou neuropraxia do nervo ulnar, que regrediu espontaneamente, e dois (9\%) apresentaram dor causada pelo material de síntese no olécrano, apresentando melhora após sua retirada. O material de síntese proeminente na região do olécrano, causador de dor, é descrito na literatura como uma possível complicação, e o índice de complicações observado neste estudo é semelhante ao encontrado na literatura, que varia de 4 a $49 \%^{2,3,4,12}$.

Não houve diferença estatisticamente significativa quando analisamos a correlação entre mobilidade articular com os tipos de fraturas A e C ( $\mathrm{p}<0,05)$; contudo, como a diferença entre o número de pacientes com fraturas do tipo A e C foi grande (4 e 18), não podemos concluir que ela foi estatisticamente significativa.

Dessa maneira, o tratamento cirúrgico das fraturas supra e intercondilianas do úmero apresentaram resultados funcionais satisfatórios em $68,22 \%$ dos casos (entre excelentes e bons), utilizando o princípio de redução anatômica com estabilidade absoluta articular e fixação das duas colunas com placas em posição ortogonal.

\section{Referências}

1. Benegas E, Ferreira Filho AA, Zoppi Filho A, Ferreira Neto AA, Negri JH, Abraão $\mathrm{F}$. Tratamento cirúrgico das fraturas intercondilianas do úmero com osteossíntese estável e mobilização precoce. Rev Bras Ortop 2000;35(9):352-7.

2. Robinson CM, Hill RM, Jacobs N, Dall G, Court-Brown CM. Adult distal humeral metaphyseal fractures epidemiology and results of treatment. $J$ Orthop Trauma 2003;17(1):38-47.

3. Webb LX. Distal Humeral Fractures in Adults. J Am Acad Orthop Surg 1996;4(6):336-44.

4. Kundel K, Braun W, Wieberneit J, Rüter A. Intraarticulardistal humerus fractures. Factors affecting functional outcome. Clin Orthop Relat Res 1996; 332:200-8.

5. Jupiter JB. Complex fractures of the distal part of the humerus and associated complications. Instr Course Lect 1995;44:187-98.

6. Jupiter JB, Morrey BF. Fractures of the distal humerus in the adult. In: Morrey BF. Elbow and its disorders. 2nd ed. Philadelphia, Saunders; 1993. p. 328-66.

7. Checchia SL, Santos PD, Miyazaki AN, Fregoneze M, Silva LA, Gomi F et al. Avaliação dos resultados do tratamento cirúrgico das fraturas supra e intercondilares do úmero em pacientes acima de 60 anos. Rev Bras Ortop 2003;38(11/12):667-75.

8. Müller ME, Allgower M, Schneider R, Willenegger H. Manual of Internal Fixation. Technique Recommended by the AO/ASIF Group. Berlin, SpringerVerlag; 1970. p. 177

9. Jupiter JB, Neff U, Holzach P, Allgöwer M. Intercondylar fractures of the humerus. An operative approach. J Bone Joint Surg Am 1985;67:226-39.

10. Gustilo RB, Anderson JT. Prevention of infection in the treatment of one thousand and twenty-five open fractures of long bones: retrospective and prospective analyses. J Bone Joint Surg Am 1976;58:453-8.

11. Bussab WO, Morettin PA. Estatística Básica. 4a ed. São Paulo: Atual; 1987. p. 321.

12. Pajarinen J, Bjorkenheim JM. Operative treatment of types $C$ intercondylar fractures of the distal humerus: results after a mean follow-up of 2 years in a series of 18 patients. J Shoulder Elbow Surg 2002;11(1):48-52.

13. McKee MD, Wilson TL, Winston L, Schemitsch EH, Richards RR. Functional outcome following surgical treatment of intra-articular distal humeral fractures through a posterior approach. J Bone Joint Surg 2000;82$A(12): 1701-7$

14. Russell GV Jr, Jarrett CA, Jones CB, Cole PA, Gates J. Management of distal humerus fractures with minifragment fixation. J Orthop Trauma 2005; 19(17):474-9.

15. Srinavasan K, Agawal M, Matthews SJE. Fractures of the distal humerus in elderly: is internal fixation the treatment of choice? Clin Orthop Rel Res 2005;(434):222-30.

16. Frankle MA, Herscovici D Jr, Di Pasquale TG, Vasey MB, Sanders RW. A Comparison of open reduction and internal fixation and primary total elbow arthroplasty in treatment of intraarticular distal humerus fractures in women older than age 65. J Orthop Trauma 2003;17(7):473-80.

17. Morrey BF, An KN, Chao EYS. Functional evaluation of the elbow. In: Morrey BF. The elbow and its disorders, 2nd ed. Philadelphia: Saunders; 1993. p. 86-97.

18. Cassebaum WH. Open reduction of $T \& Y$ fractures of the lower end of the humerus. J Trauma 1969:9(11):915-25.

19. Wilkinson JM, Stanley D. Posterior surgical approaches to the elbow: a comparative anatomic study. J Shoulder Elbow Surg 2001;10(4):380-2.

20. Schildhauer TA, Nork SE, Mills WJ, Henley MB. Extensor mechanismsparing paratriciptal posterior approach to the disal humerus. J Orthop Trauma 2003;17(5):374-8.

21. McKee MD, Wilson TL, Winston L, Richards RR, Schemitsch EH. Elbow extension strength following treatment of intra-articular distal humeral fractures. A comparison of triceps splitting versus olecranon osteotomy approaches. J Orthop Trauma 1999;13(4):279-80.

22. Korner J, Diederichs G, Arzdorf M, Lill H, Josten C, Schneider E et al. A biomechanical evaluation of methods of distal humerus fracture fixation using locking compression plates versus conventional reconstruction plates. J Orthop Trauma 2004;18(5):286-93. 